\title{
EXTENDED STABILITY MARGINS ON CONTROLLER DESIGN FOR NONLINEAR INPUT DELAY SYSTEMS
}

\author{
Otto J. Roesch, Hubert Roth, Asif Iqbal \\ Institute of Automatic Control Engineering \\ University Siegen, Germany \\ \{otto.roesch, hubert.roth\}@uni-siegen.de \\ iqbal@ipp.zess.uni-siegen.de
}

\begin{abstract}
In this paper, upper bounds for time-varying delays in the real-time control of a lab helicopter in the sense of practical stability analysis are derived. Sub-optimal controller parameters for this non-linear plant with a given maximum time-delay are calculated with the help of Lyapunov-Razumikhin method using LMIs (Linear Matrix Inequalities). These parameters are then optimized for extended time-delay margins by considering design specifications like settling-time and overshoot, using a novel 3dimensional search algorithm in the vicinity of the previously identified values. Trade-off between settling-time and overshoot is achieved with the help of appropriate weighting factors in an objective function based approach. Copyright (C) 2005 IFAC
\end{abstract}

Keywords: Time delay, Lyapunov Stability, Remote Control, State Space Realization, Nonlinear Analysis

\section{INTRODUCTION}

The presence of time delays in industrial processes is an important phenomenon and must be considered in the stability analysis and controller design. Delays could occur because of data communication or by material flow in machines. They can be classified as constant or time-varying, bounded or unbounded, distributed or not, deterministic or stochastic. Control of these time-delay systems is an active research area. Normally the delay may appear either in the state, or in the input (or output) of the corresponding systems (Marshal, 1979). Stability of such systems is the most important property to be considered and becomes hard to establish keeping in mind the fact that introduction of time-delay in the states or input causes the system to have infinite number of poles or zeros (Marshall, 1992) for closed loop systems. The reported stability could either be delay-independent or delay-dependent, the former being more restrictive than the later and more difficult to ensure (Boukas, 2002).

In this paper, signal delays due to data-transmission are considered, which could occur e.g. by large/long bus-system or by the use of the internet (with TCP/IP or UDP as protocols). If the control-system and the plant are spatially separated, the delays occur two times in the control loop, once from the plant to the controller (forward direction), and secondly from the controller back to the plant (backward direction), see Fig. 1. Since time delays in the control loop have a major influence on stability of control systems, they must be carefully considered in the mathematical description of the system dynamics. Zhang et al. (2001) gives an excellent overview of stability issues in networked control systems. They divide the stability issue into two categories, i) developing new network protocols for control applications, and ii) to take the network as it is, and focus on control algorithms to achieve stability. For a general class of systems, the authors suggest to mark stability regions using simulations while continuing to increase the time delay. In our work, we first find a conservative controller using the Razumikhin theorem, and then we go further and receive with a simulation setup optimal control parameters for a stable control system. 
This contribution is based on the work by Niculesu et. al. (1997), the delay-dependent stability with an input delay of a twin rotor laboratory helicopter is considered. For a remote control, two kinds of strategies can be considered for such a plant. In the first one, a control law is designed remotely and is sent to the plant over some communication link, which is executed locally on the plant. In this case there is no input delay in the system and hence no bounds on delays. The only condition is that the system must be stabilizable. In the second case, the output of the controller towards the plant is delayed. This causes an input delay and thus introducing the concept of stability margins based on time-delay, which will be the focus of this work.

An LMI (Linear Matrix Inequality) based approach is used to find the parameters of a third-order statefeedback controller. A Lyapunov-Razumikhin function maximizes the time-delay (in sub-optimal sense) of the closed loop system for a stable system like the one considered in Fig. 1. Once the stability of this sub-optimal controller is achieved, it becomes of practical interest to see how much the parameters of this stabilizing controller can be varied while remaining within the stability margin of the system. Furthermore, can the upper bound on the input delay be improved for such a perturbation? These questions will be dealt with in detail within the following sections. In addition, specific consideration on the performance of the plant, such as settling-time, overshoot, etc., can also be taken care of in the design of the controller as will be shown later.

\section{PROBLEM DEFINITION}

In contrast to systems free of delays, a time-delay system considers not only the current system states, but also the past states which lead to a system described by delay differential equations (DDE):

$$
\dot{x}(t)=f(t, x(t), x(t-\tau)),
$$

with $x \in \mathbb{R}^{n}, \tau>0$. And appropriate initial conditions derived on the interval $\left[t_{0}-\tau, t_{0}\right]$. Due to the tremendously increasing usage of tele-control over the internet, such time delay control systems get more and more important in practical applications. A general representation is shown in Fig. 1, with $K$ as the controller, $G_{s}$ the plant, w the desired input and $x$ the plant output. The time delay occurs from the controller to the plant and again from the plant backward to the control unit.

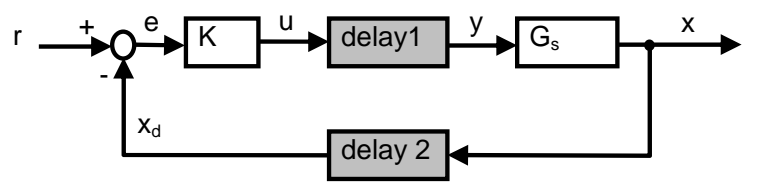

Fig. 1. Closed loop system with time-delays in the communication channels
In general, control design algorithms do not consider time delay aspects in the control loop, like root-locus, Nyquist plots or pole placement approaches. Stability analysis of time delay systems are easier to handle in the time domain than in the frequency domain, especially in the case of time-varying uncertainties and nonlinearities (Gu, 2003). Based on the second Method of Lyapunov, the theorems such as the Krasovskii and the Razumikhin provide stability conditions for time-delay systems in the time-domain. These methods deal exclusively with state space descriptions and the stability tests amount to sufficient conditions that can be posed as solutions of LMI problems.

These robust control approaches are very conservative and have large stability margins, as it was eventually experimentally tested. Based on a controller, calculated with the Razumikhin theorem, a 3-D search algorithm is imposed for further improving the system response.

\section{DEFINITION OF THE LYAPUNOV- RAZUMIKHIN FUNCTION}

The Razumikhin theorem is an extension to the classical Lyapunov stability theorem. It is used in the derivation of the following controller design by Niculescu, et al. (1997), the definition can be found in (Hale, et al., 1993) or (Gu, 2003).

\subsection{Solution Approach - Controller Algorithm}

Based on a system presented in state space form, we can derive the equation with an input delay of the following form:

The system $\dot{x}(t)=A x(t)+B u(t)$ considers the following input $u(t)=K x(t-\tau(t))$ as a control law. This results in a closed loop description to $\dot{x}(t)=A x(t)+B K x(t-\tau(t))$. Applying the Razumikhin theorem for such an input delay system, the following theorem found in Niculescu, et al. (1997), results in:

If the following inequalities hold simultaneously

$$
\begin{aligned}
& {\left[\begin{array}{c}
\frac{1}{\tau *}\left[\begin{array}{c}
Q A^{T}+A Q \\
+B W+W^{T} B^{T}
\end{array}\right] \\
+\left(\beta_{1}+\beta_{2}\right) Q \\
W^{T} B^{T}
\end{array}\right]<0} \\
& -\beta_{1} Q+A Q A^{T} \leq 0
\end{aligned}
$$




$$
\beta_{2}\left[\begin{array}{cc}
-Q & 0 \\
0 & 0
\end{array}\right]+\left[\begin{array}{cc}
0 & B W \\
W^{T} B^{T} & -Q
\end{array}\right] \leq 0
$$

with $Q \in \mathbb{R}^{n \times n}$ being positive-definite and symmetric, a matrix $W \in \mathbb{R}^{m \times n}$ and the positive scalars $\beta_{1}$ and $\beta_{2}$ hold, then the closed loop system is uniformly asymptotically stable. The input of the form

$$
u(t)=W Q^{-1} x(t-\tau(t))
$$

for all the delays $\tau(t)$ satisfies $0 \leq \tau(t) \leq \tau$ * with $K=W Q^{-1}$ is a substituting controller. For a complete proof see the excellent paper of Niculescu, et al. (1997).

\subsection{Controller Calculation}

In order to solve inequalities (2), (3) and (4), it is necessary to convert the bilinear conditions into a LMI system, based on the algorithm proposed by Niculescu, et al. (1997). (This is necessary because the variables $\beta_{1}$ and $\beta_{2}$ are multiplicatively associated with $Q$, and therefore not solvable with available LMI solvers).

To solve a so called generalized eigenvalue problem (gevp), like equation (2), a scalar (here: $\lambda=1 / \tau^{*}$ ) gets minimized by holding the set of inequalities. The optimization variable must be an isolated outer-factor of a LMI-matrix. LMI-Solver (e.g. LMI-Toolbox in Matlab) can not handle equation (2) directly. It must be first converted into the generalized eigenvalue problem (gevp) form, whereas the LMI of equation (2) yields to

$$
\begin{gathered}
{\left[\begin{array}{cc}
\left(\beta_{1}+\beta_{2}\right) Q & B W \\
W^{T} B^{T} & -\frac{1}{2} Q
\end{array}\right]<\underbrace{\left[\begin{array}{cc}
Y & 0 \\
0 & 0
\end{array}\right]}_{[\text {] }>0 \text { defined! }}} \\
Y<\lambda(-1)\left(Q A^{T}+A Q+B W+W^{T} B^{T}\right) \\
-\left(Q A^{T}+A Q+B W+W^{T} B^{T}\right)>0
\end{gathered}
$$

with $\lambda=\frac{1}{\tau^{*}}$.

Now the upper bound for the time delay $\tau *$ can be maximized by minimizing $\lambda$.

Algorithm from Niculescu, et al. (1997), to solve the nonlinear gevp problem

- Step one:

Solve equation (8) with respect to $Q>0$.

- Step two:

Solve the LMI system (equations (3)(4)(6)(7)(8)) whereas $Q$ is fixed.

$$
\left\{\begin{array}{cc}
\min _{W, \beta_{1}, \beta_{2}} \lambda\left(W, \beta_{1}, \beta_{2}\right) & \text { s.t. } \\
(3),(4),(6)-(8) & \text { hold for } \mathrm{Q}>0
\end{array}\right.
$$

- Step three:

Solve the LMI system (equations (3)(4)(6)(7)(8)) whereas $\mathrm{W}, \beta_{1}$ and $\beta_{2}$ are fixed.

$$
\left\{\begin{array}{lc}
\min _{Q>0} \lambda(Q) & \text { s.t. } \\
(3),(4),(6)-(8) & \text { hold for fixed } \mathrm{W}, \beta_{1}, \beta_{2}
\end{array}\right.
$$

Return to step 2 until the convergence of $\lambda$ is attained within a desired precision.

\section{HELICOPTER SYSTEM}

The system under consideration is represented by a helicopter model from Quanser (2004), see Fig. 2. It consists of a fixed base, on which a rotary arm is mounted. The arm carries the helicopter body on one end, and a counterweight on the other. The arm can make an elevation motion around the angle epsilon. An encoder mounted on the axis allows measuring the elevation angle of the arm. The helicopter body is fixed at one end of the arm and is free to elevate in a certain range. Two motors with propellers mounted on the helicopter arm can generate a force proportional to the voltage applied to the motors. The force generated by the propellers cause the helicopter body to lift off the ground. The purpose of the counterweight is to reduce the power requirements on the motors around equilibrium. The corresponding nonlinear mathematical model looks like the following:

$$
J_{g e s} \cdot \ddot{\varepsilon}=-g \cdot y \cdot(M+m) \cdot \sin (\varepsilon)+2 \cdot k_{t} \cdot r \cdot v
$$

The dimension parameters can be seen in Fig. 2, where $k_{t}=0.5 \mathrm{~N} / V$ represents the motor constant and $v$ the voltage to the motors, $g$ the gravity constant and $J_{g e s}$ the moment of inertia around the rotating point, $m$ is the mass of the helicopter blades inclusive motors and the fixing devices, $M$ is the counterweight. Please note, that if $\dot{y}(t)$ is not available, we can construct a "classical" (asymptotic) observer or to approximate $\dot{y}(t)$ by some delay terms $\dot{y}(t) \approx \frac{y(t)-y(t-\theta)}{\theta}$, as suggested by Niculescu and Michiels (2004) for a different control problem.

To apply the Lyapunov-Razumikhin control algorithm presented in the previous section, the model must be linearized around the quiescent point $\varepsilon=0$ and transferred into the state space form

$$
\begin{aligned}
& \dot{x}=A x+B u \\
& y=C x
\end{aligned}
$$




$$
x=\left[\begin{array}{c}
\dot{\varepsilon} \\
\varepsilon \\
\int \varepsilon
\end{array}\right]
$$

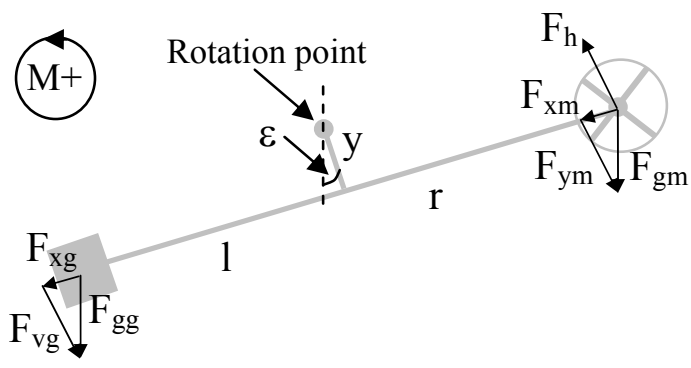

Fig. 2. Schematic drawing of the 3D Helicopter model with fixed travel and pitch axis

A third state was added to represent the integral of the elevation angle, which is necessary to reach steady state accuracy of the elevation angle. Parameter identification enabled the analysis of the damping coefficient for an improvement of the mathematical model. This was done by the actuation of a step input to the real system while the decaying oscillation was observed, for which a damping factor got derived. The corresponding numerically computed state space model results finally in:

$$
\begin{aligned}
& A=\left[\begin{array}{ccc}
-0.08 & -2.97 & 0 \\
1 & 0 & 0 \\
0 & 1 & 0
\end{array}\right] ; \quad B=\left[\begin{array}{c}
0.555 \\
0 \\
0
\end{array}\right] \\
& C=\underline{I}_{3} ;
\end{aligned}
$$

By applying the Razumikhin control algorithm from the previous section, the following control vector is obtained

$$
K=\left[\begin{array}{lll}
-3.155 & -2.022 & -3.637
\end{array}\right],
$$

for a maximum time delay of $\tau^{*}=0.116$ seconds. By applying this control vector to the real plant, we can see that the controller is very conservative and shows even a stable behaviour for time delays up to 0.4 seconds. The analysis of the stability margin on the controller design was necessary for a further improvement of the system, even for small delays.

\section{NONLINEAR SIMULATION DEVELOPMENTS}

For a stability margin extension, a simulation of the nonlinear mathematical model of the helicopter is set up in Simulink. The above calculated controller parameters from the Razumikhin approach are used as a starting point. By varying these controller parameters successively with additional gain factors and considering the settling time and the overshoot, a further improvement of the control system is derived. These additional gain factors were increased from 0.1 to 3 sequentially to consider all possible combinations (this range of the gains was previously analysed). The Matlab Simulink simulation block diagram is given in Fig. 3.

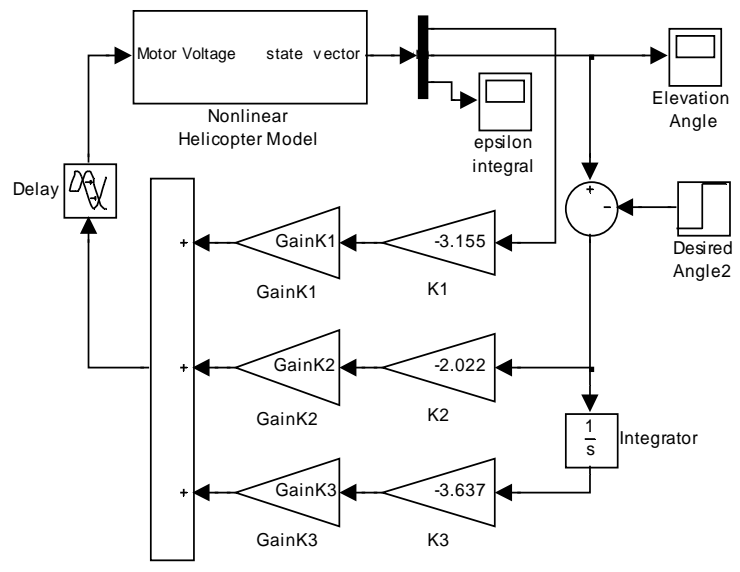

Fig. 3. Simulink simulation for the stability margin extension with varying additional gain factors with the nonlinear helicopter model

As shown in Fig. 3, the additional gain parameters (GainK1, GainK2 and GainK3) are multiplied with the parameters from (15). The "Elevation Angle" is considered for the analysis of the settling time and overshoot. Delay was fixed for each pass-through to get the best controller parameters.

\section{RESULTS}

The simulation results obtained in section 5 are shown in Table 1, where the delay is varied in steps from 0.1 to 0.7 seconds, with an increment of 0.1 seconds. It must be noted that in obtaining these parameters, the objective is to minimize the settling-time for a given value of time delay. Settling times and overshoots against the gains are plotted in Fig. 4. System response in terms of elevation angle for $\tau=0.4 \mathrm{sec}$ is shown in Fig. 5.

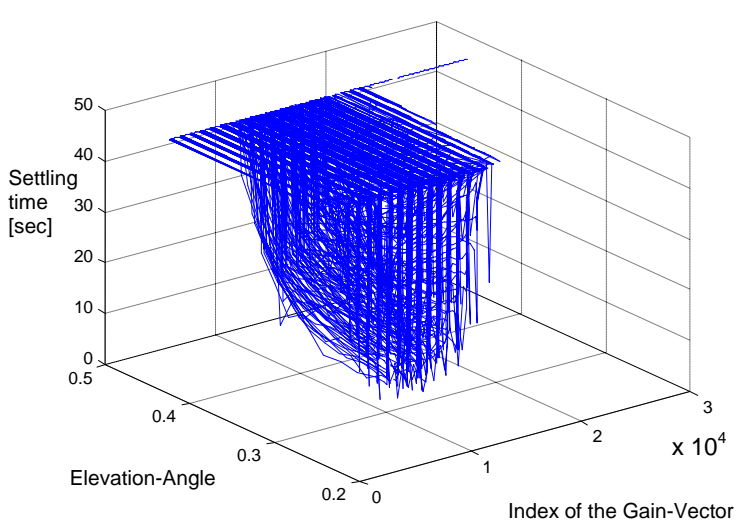

Fig. 4. Settling time and elevation angle vs. gains, for an elevation step-input of $0.25 \mathrm{rad}$ 


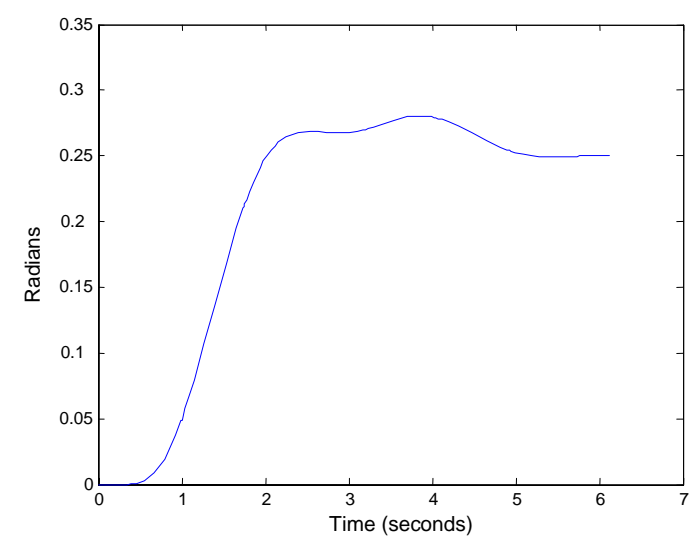

Fig. 5. Elevation angle system response example with minimal settling time for a constant delay of $\tau=0.4$ seconds

Careful examination of the data in Table 1 reveals a steady decrease in the gain parameters for increasing time delays. This can be explained in the context of passivity theory. Lowering the gains on control inputs is equivalent to dissipating the additional energy induced into the system by time-delays (Anderson and Spong, 1989). At the same time, we see an increase in the settling times for increasing delays which is quite normal. It has to be noted that the Razumikhin theorem provides the gain parameters for time-varying delays so the system will remain stable with given gain values even for time-varying delays as far as the delay remains bounded by a certain given value.

In order to obtain a continuous range of gains for intermediate values of delays (in the range from 0 to 0.7 seconds), the identified values of gains are linearized afterwards, as shown by the plot in Fig. 6 where the additional gain parameter GainK1 is displayed. The dots are the actual values, produced by the simulation, whereas the solid line is obtained by linear curve fitting, providing gains that are finally used in the real-time implementation, which shows good performance on actual non-linear plants.

Table 1: Simulation results for the additional GainKx parameters

\begin{tabular}{llllll}
$\begin{array}{l}\text { Time } \\
\begin{array}{l}\text { Delay } \\
(\mathrm{sec})\end{array}\end{array}$ & $\begin{array}{l}\text { Min. } \\
\text { Settling } \\
\text { Time } \\
(\mathrm{sec})\end{array}$ & $\begin{array}{l}\text { Max. } \\
\text { Over- } \\
\text { shoot } \\
(\mathrm{rad})\end{array}$ & $\begin{array}{l}\text { Gain } \\
\text { K1 }\end{array}$ & $\begin{array}{l}\text { Gain } \\
\text { K2 }\end{array}$ & $\begin{array}{l}\text { Gain } \\
\text { K3 }\end{array}$ \\
\hline 0.1 & 4.2514 & 0 & 1.8 & 2.3 & 1.5 \\
0.2 & 2.7529 & 0.0043 & 1.6 & 2.5 & 1.8 \\
0.3 & 3.6228 & 0.0015 & 1.8 & 1.7 & 1.2 \\
0.4 & 6.1138 & 0.0302 & 1.2 & 0.6 & 1.3 \\
0.5 & 14.998 & 0 & 0.9 & 0.1 & 0.4 \\
0.6 & 31.412 & 0 & 0.4 & 0.1 & 0.2 \\
0.7 & 80.063 & 0 & 0.3 & 0.1 & 0.1 \\
\hline
\end{tabular}

Furthermore, if it is desired to improve the overshoot behavior in the response of the system, it can be achieved by first constructing an objective function that gives weight to the overshoot in addition to the settling time. This objective function is then evaluated over the whole space of admissible gain parameters. Minimum value of this objective function will provide the optimal gain parameters while satisfying the performance requirements.

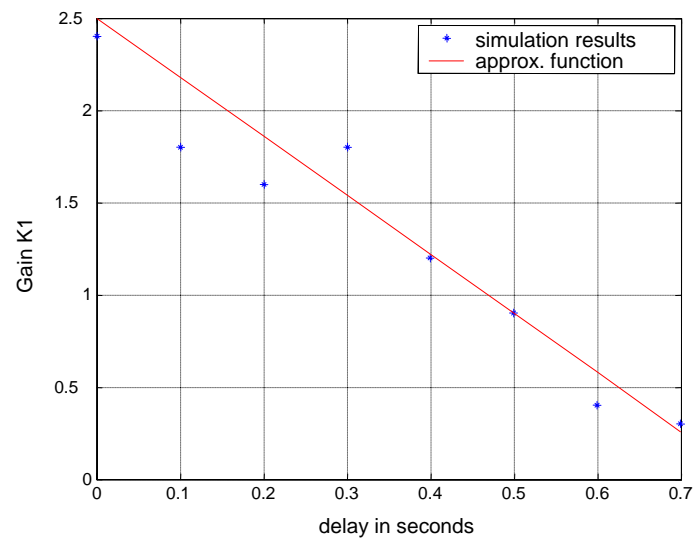

Fig. 6. Linearization of the additional gain parameters, GainK1 is shown here, with a delay of 0 to 0.7 seconds.

The following expression is used as the objective function to be minimized

$$
f\left(t_{s}, \theta_{O S}\right)=\alpha \cdot t_{s}+\beta \cdot \theta_{\text {oS }},
$$

where $t_{s}$ and $\theta_{O S}$ are settling-time and overshoot respectively. $\alpha$ and $\beta$ are weighting factors.

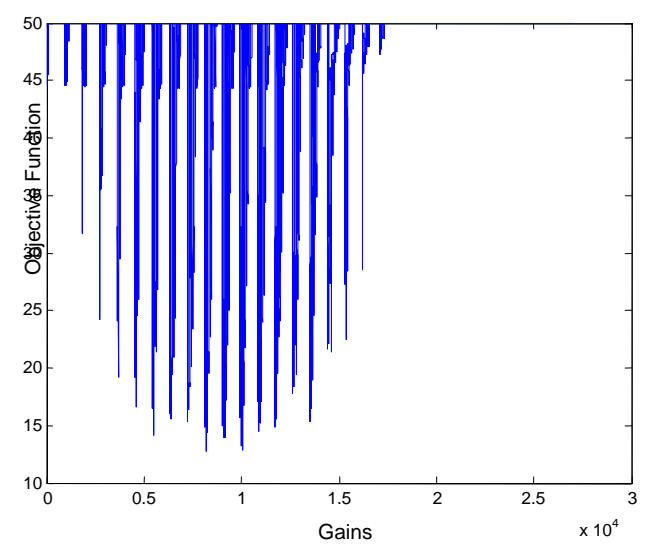

Fig. 7. Objective function $f\left(t, \theta_{o S}\right)$ vs. Gains

If the desired function gives $75 \%$ weight to settling time and $25 \%$ to overshoot then $\alpha=0.75$ and $\beta=0.25$. Applying this performance requirement to the case where $\tau=0.4, \tau$ being the time delay, and conducting a search over admissible controls, one gets new values for the settling time and the overshoot

$$
\begin{aligned}
& t_{s}=6.7292\left(t_{s, \text { old }}=6.1138\right) \\
& \theta_{O S}=0.0125\left(\theta_{\text {OS, old }}=0.0302\right)
\end{aligned}
$$

These values indicate that a $10 \%$ increase in settlingtime can be traded-off against a $58.75 \%$ decrease in 
overshoot. Previous and new gains obtained through the weighted objective function in (16) are given below:

$$
\begin{aligned}
& K_{\text {new }}=\left[\begin{array}{lll}
1.0 & 0.4 & 1.0
\end{array}\right] \\
& K_{\text {old }}=\left[\begin{array}{lll}
1.2 & 0.6 & 1.3
\end{array}\right]
\end{aligned}
$$

A plot of the objective function vs. gains is shown in Fig. 7.

\section{CONCLUSIONS}

In this work, the Lyapunov-Razumikhin approach is used to identify sub-optimal controller parameters for a nonlinear delayed input system while maximizing the time-delay and ensuring the stability. These controller parameters are then optimized using a combination based on 3D search in order to achieve certain performance requirements like settling time and overshoot. The delay bounds given by Razumikhin theorem are extended using our approach in the sense of practical stability.

An approach based on weighted objective function is further used to achieve a performance balance between minimum settling time and maximum overshoot allowed.

The search algorithm presented uses combinations of parameter values to find the optimal values with an increment of 0.1 in the space of admissible gains. Fig. 7 show that we are able to find the global minimum in terms of settling times. At the same time, it can be concluded that even more accurate parameters can be obtained if the incremental step size is reduced, which becomes demanding in terms of computational requirements. For future work, it is therefore suggested to use different heuristic approaches like genetic algorithm and simulated annealing to optimize the search for controller parameters.

For a practical usage, the above presented procedure for the derivation of a controller must be automated in some way. For example, by specifying the transfer function and the maximum time delay (which could occur in the system), a control engineer could directly get the controller. Of course it is not known a priori if a stable controller for the specified maximum time delay can be found by the presented algorithm as it depends on the plant and the maximum delay of the system. The function for approximation of gain parameters based on input delay (see Fig. 6) is only a linear function. Experimental results show that it can further be improved by using a higher order function. The approach described in this work, makes the following assumption for signal RTT (Round Trip time), as is generally done in network control systems literature $R T T=$ delay $1+\operatorname{delay} 2$ (see Fig. 1).

\section{REFERENCES}

Anderson, R.J.; Spong, M.W. (1989). Asymptotic stability for force refecting teleoperators with time delay. Proceedings of the IEEE International Conference on Robotics and Automation, pages 1618-1625.

Boukas, E.-K.; Liu, Z.-K. (2002). Deterministic and Stochastic Time Delay Systems, Control Engineering, Birkhaeuser

Gu, K.; Kharitonov , V.L.; Chen, J. (2003). Stability of Time-Delay Systems, Birkenhäuser, 2003

Hale, J.K.; Renardy Y.; Wheeler, R.L. (1993). Introduction to functional differential equations, In: Volume 99 of Applied Mathematical Sciences, Springer-Verlag.

Marshall J.E.; Gorecki H.;,Korytowski A.; Walton, K., (1992). Time Delay Systems, Stability and Performance Criteria with Applications, Ellis Horwood Press.

Marshall, J.E. (1979). Control of time-delay systems, Peter Peregrinus, Ltd.

Niculescu, S.I.; Michiels, W. (2004). Stabilizing a Chain of Integrators Using Multiple Delays, IEEE Transactions on Automatic Control, Vol.49, No.5.

Niculescu, S.-I.; Fu, M.; Li, H. (1997). Delay Dependent Closed Loop Stability of Linear Systems with Input Delay: An LMI Approach, Proceedings of the 36th Conference on Decision \& Control, San Diego, California, USA

Quanser (2004) 3D Helicopter Experiment with DAQ Board and WinCon Software, www.quanser.com.

Zhang, W.; Branicky, M.S.; Phillips , S.M. (2001) Stability of Networked Control Systems, IEEE Control Systems Magazine. 